\title{
Electromyographic Activity of Lower Extremity Muscles during Soccer Heading
}

\author{
Ahmed Abdel-Azeem Abdallah*, Abdel-Baset Ahmed Abdel-Haleem**
}

\begin{abstract}
:
In the world of sports, soccer is unique because of the purposeful use of the head for controlling and advancing the ball. Heading of the ball is an essential part of the game. There is little knowledge about the roles of the lower limb muscles during ball heading. In this study, we used electromyography to examine the actions of the quadriceps, tibialis anterior, hamstring and gastrocnemius muscles during ball heading by 15 young professional soccer players while standing, jumping, running or running and jumping. We found that all examined lower limb muscles were active during ball heading, especially the tibialis anterior, which produced significantly more power than other muscles in all types of heading. This may be important to consider by soccer trainers and players for both safety and heading performance.
\end{abstract}

Keywords: EMG, football, heading, lower limb muscles.

\section{Introduction:}

Coccer is a game often played along the ground but sometimes a high ball is the option that some players might choose. Once the ball is in the air, both teams will want to challenge for it. If a player wait for the ball to drop, he run the risk of losing possession, or even may be conceding a goal $(3,10)$.

Heading of a soccer ball may be defined as the intentional act of directing the soccer ball with the forehead. It is an integral skill that soccer players must master in order to participate fully in their sport. In fact, soccer is the only sport where the flight path of the object projectile is skillfully and accurately altered with the forehead. The proper technique for heading the soccer ball, as it is taught to all soccer players, is to use only the forehead region, approximately an area between the eyebrows and hairline in the vertical dimension, and between the most forward portion of the temples in the horizontal dimension (9).

In soccer, heading is purposeful and may be used as defensive, offensive. Glancing, flicking, and diving header.Defensive heading normally

* Faculty of Physical Education, Mansoura University, Egypt.

** Faculty of Physical Education, Mansoura University, Egypt. involves the most power. Offensiveheading can be used in and around opponent's goal, in order to head the ball downwards away from the goalkeeper(11). Glancing heading most often use by offensive players as they run across the goal. Flicking heading which purpose is quick redirection the ball. And, diving heading which is primarily used as offensive goal-scoring technique; however, it can be just as effective as a defensive play $(3,10)$. All types of heading can be performed from standing, running, jumping, or running/jumping. Accordingly these skills may be classified in a continuum from discrete to serial.

Soccer heading would be described as a three phases skill: the preparatory phase, main phase, and the follow through or end phase. The success of the main phase depends mainly on the success of the preparatory phase, which in turn consists of two motions; the back- and forward-swing. In heading, the back-swing motion provides more distance to absorb the force and generate an appreciable amount of time and space for subsequent forward-swing motion. $(3,4)$.

Purpusefull heading can only be done in the presence of a solid and stable base support from lower extremities muscles. In turn, good core stability will provide the lower extremities with 
a solid base during lean back and forward, run, jump, and kick.

In performing heading, the upper and lower muscles required to support force and balance during the execution (8), because power flow from feet to head. However, previous research focused on head and neck neglecting lower leg muscles. In heading the lower leg muscles are of greater importance for maintaining balance, and consider as base of support during back or forward swing, and pushing the body forward during running or upwards during jumping $(8$, 9).

Therefore, the present study focused on power output from lower limb muscles to compare the effectiveness of four pair lower limb muscles during the preparatory phase, namely: the quadriceps (QC), tibialis anterior (TA), gastrocnemius (GM), and hamstring (HS). In order to make quantitative comparisons, EMG was used to analyze RMS signals of the underlying right and left side muscles during the preparatory phase of performing four heading skills: heading from standing, running, jumping, and running/ jumping. These muscles were chosen because of their importance as superficial muscles that help to control the whole body before, during, and after ball impact.

\section{Methods:}

\subsection{Subjects}

Fifteen male soccer players (age $=14.16 \pm 0.60$ years, height $=1.56 \pm 0.04$, weight $53.67 \pm 4.01$ ) participated in this study at an offseason training session during which they were completing resistance training and conditioning program in preparation for the upcoming season. Subjects training age range between 2-3 years. All subjects denied neck injury within 6 months of the study and were free of other neurological disorders (eg. epilepsy, seizures). They also completed a health history questionnaire.

\subsection{Experimental setup}

Subjects were asked to perform four heading skills from standing, running, jumping, and running/jumping. To begin performing heading, participants were performed a neck warm-up consisting of clockwise and counter-clockwise neck rotations, neck flexion and neck extension stretching before starting their heading trials. Heading Trails were separated by at least 30 seconds (9).

Each participant performed four heading skills in four sessions, one per day. Heading Skills were performed in accordance with the following order: from standing, jumping, running, running/jumping, in that order. Each skill was performed until the subject recording a successful trial which was defined as making ball contact with the forehead; and pulling arms back.

\subsection{Electromyography (EMG)}

EMG measurements and analysis of data were done in the kinesiology lab at College of physical education - Mansoura University. The EMG signals were obtained from four right and left of QC, HS, TA, and GM during the initiation phase, before head impact, for four heading skills (standing, running, jumping, running/jumping). Eight channels Wireless EMG surface electrodes were used (Delsys Inc. Type DE-02.3, Boston, MAUSA). Electrode placement wasconducted in accordance with standardpractice of each heading skill. After skin preparation (shaving, gently scrubbing, and cleaning with alcohol), the bipolar surface electrodes [DE 2.1 Single Differential Surface EMG Sensor, Delsys, Inc., Boston MA, USA; Sensor Contacts -2 silver bars, $10 \mathrm{~mm}$ long $\times 1$ $\mathrm{mm}$ diameter; Contact Spacing - $10 \mathrm{~mm}$; CMRR - $92 \mathrm{~dB}$ (typical), $84 \mathrm{~dB}$ (minimum)] were placed over the mid-bellies of the designating muscles.Muscle activity was collected simultaneously before the instans of ball impact.The sampling rate was at $1000 \mathrm{~Hz}$, bandwidth $20-450 \mathrm{~Hz}$. 92dB (typical) common mode rejection ratio (input impedance $>10$ $\mathrm{M} \Omega$ ). Single Differential, dimensions $41 \times 20$ x $5 \mathrm{~mm}$, noise $1.2 \mathrm{uV}$ (RMS, R.T.I.), Delsys Inc, Boston, MA) and recorded on an EMG Myomonitor IV Wireless Transmission \& Datalogging System (Delsys Inc, Boston, MA), with real-time EMG viewing on a laptop computer. The raw EMG signal was converted to a root mean square (RMS) at each 0.0625 second interval during the preparatory phaseof heading before ball impact.

\subsection{Statistical Analysis}


Data were analyzed using descriptive statistics. Paired t-test was used to compare the RMS between right and left side muscles. Analyses of variance (ANOVA) were conducted to compare RMS values of the perspective muscles in each heading Tukey test was used when appropriate. Alpha levels of 0.05 were used to test for significance and SPSS for Windows (version 17.0) was used for data analysis.

\section{Results:}

The overall EMG-RMS mean values for right and left side muscles in each heading skill is shown in Figure (1), results indicated gradually decrease in effort values among the four heading skills as follows: running/jumping, jumping/ running, standing, respectively. And, effort exerted by left side muscles were larger than that of the right side muscles. The comparisons between these values showed significant increase in the RMS of left side muscles in comparison to the right side for heading from jumping $(\mathrm{p}=0.015)$, and running/jumping $\quad(\mathrm{p}=0.000)$, while no significant difference was found between both sides for performing heading from standing and running.

Figure (1)

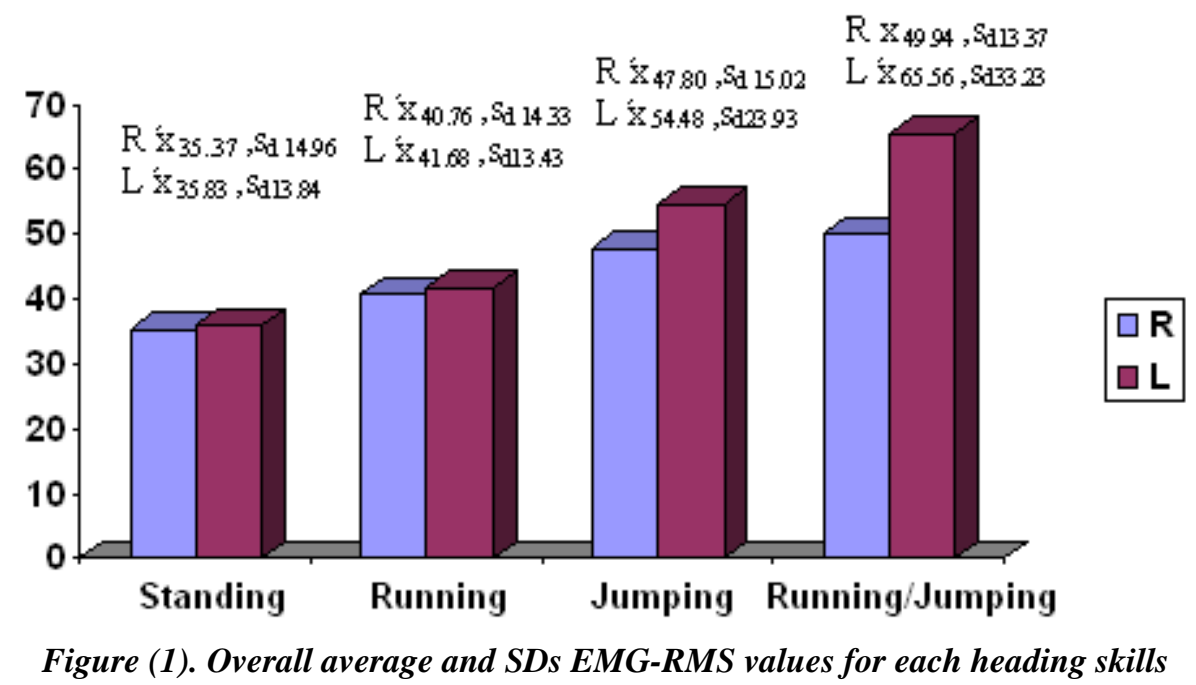

Concerning the EMG-RMS average values and SDs regarding right and left side muscles for each heading skill, results are shown in table 2 .

Generally, these results indicated different values among the four designated muscles for each heading skill (Figure 2.).
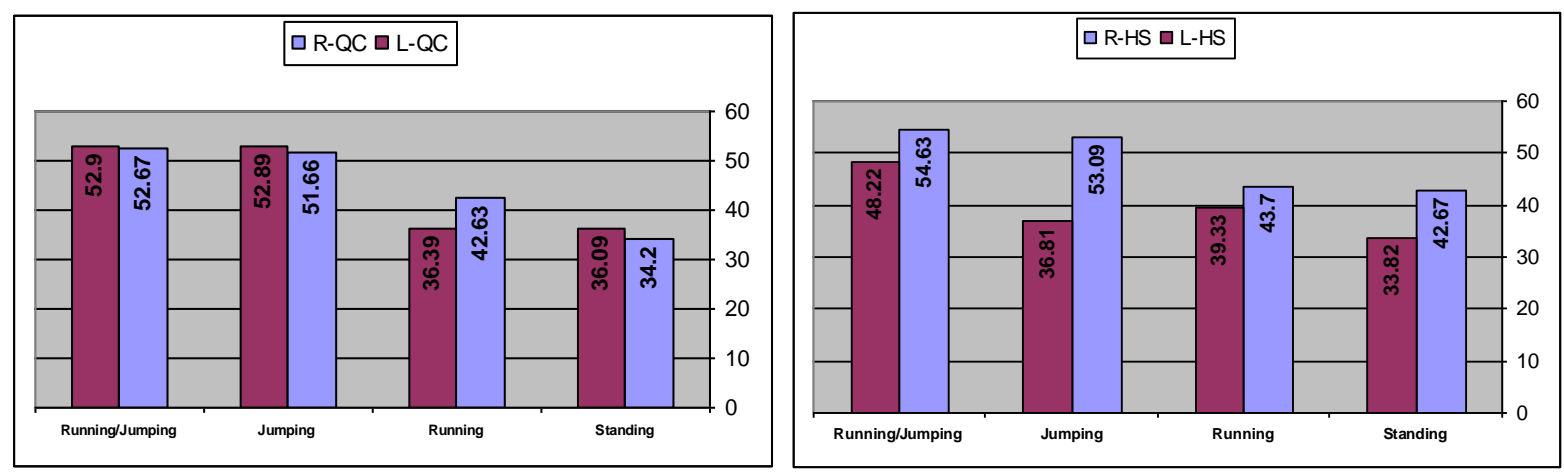

Figure (2). Average and \pm SD values of EMG-RMS power values for each muscle among the four heading skills

Regarding differences existed among the EMGRMS values concerning the designated muscle for each heading skill, ANOVAs results revealed significant difference for either the right or left side muscles $(\mathrm{P}=0.000)$, except for right side muscles in jumping $(\mathrm{P}=0.003)$ (Table. 3 ), therefore, Tukey tests were conducted to subscribe where significant(s) exsists (Table 4.). 
Table 3: One way ANOVA among the RMS amplitude of the four muscles for each heading skill
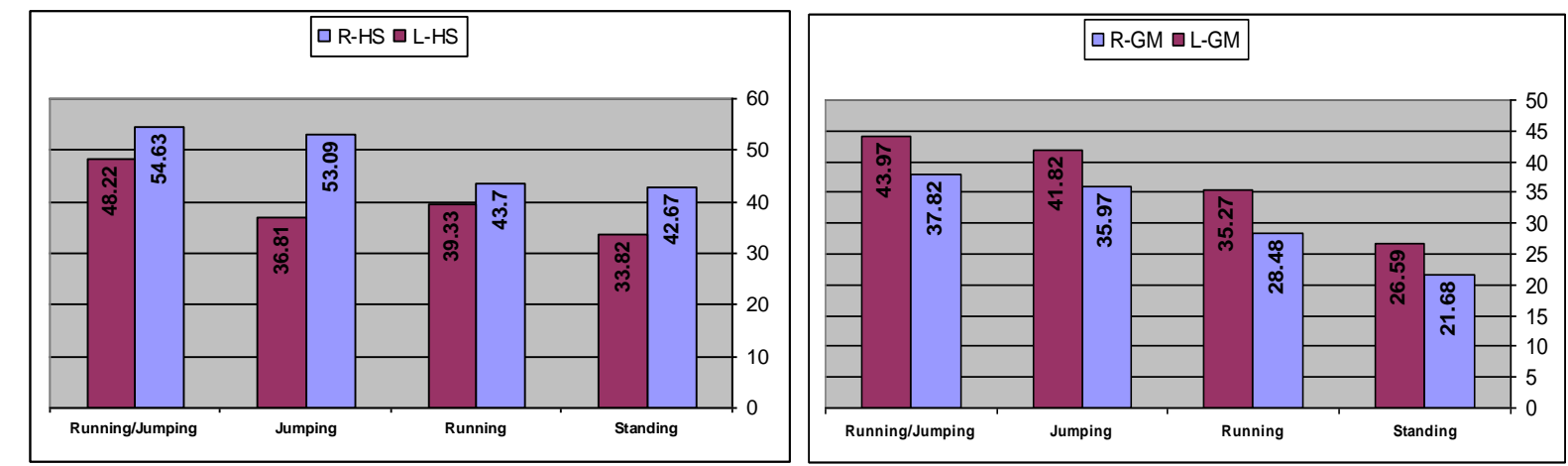

\begin{tabular}{|c|c|c|c|c|c|c|c|}
\hline Heading & SideMuscle & Source & Sum of Squares & Df & Mean Square & $\mathrm{F}$ & Sig. \\
\hline \multirow{4}{*}{ Standing } & \multirow{2}{*}{ Right } & Between muscles & 4489.623 & 3 & 1496.54 & 9.627 & 0.000 \\
\hline & & Within muscles & 8705.131 & 56 & 155.45 & & \\
\hline & \multirow{2}{*}{ Left } & Between muscles & 3150.886 & 3 & 1050.30 & 7.207 & 0.000 \\
\hline & & Within muscles & 8161.080 & 56 & 145.73 & & \\
\hline \multirow{4}{*}{ Running } & \multirow{2}{*}{ Right } & Between muscles & 3278.670 & 3 & 1092.89 & 6.923 & 0.000 \\
\hline & & Within muscles & 8839.857 & 56 & 157.86 & & \\
\hline & \multirow{2}{*}{ Left } & Between muscles & 4072.814 & 3 & 1357.61 & 11.564 & 0.000 \\
\hline & & Within muscles & 6574.253 & 56 & 117.40 & & \\
\hline \multirow{4}{*}{ Jumping } & \multirow{2}{*}{ Right } & Between muscles & 2850.974 & 3 & 950.33 & 5.088 & 0.003 \\
\hline & & Within muscles & 10459.534 & 56 & 186.78 & & \\
\hline & \multirow{2}{*}{ Left } & Between muscles & 22774.394 & 3 & 7591.47 & 38.649 & 0.000 \\
\hline & & Within muscles & 10999.456 & 56 & 196.42 & & \\
\hline \multirow{4}{*}{ Running/Jumping } & \multirow{2}{*}{ Right } & Between muscles & 3004.025 & 3 & 1001.34 & 7.442 & 0.000 \\
\hline & & Within muscles & 7535.443 & 56 & 134.56 & & \\
\hline & \multirow{2}{*}{ Left } & Between muscles & 53802.426 & 3 & 17934.14 & 88.406 & 0.000 \\
\hline & & Within muscles & 11360.276 & 56 & 202.86 & & \\
\hline
\end{tabular}

Results of Tukey test indicated that these significants were not alike for right and left side muscles. Significant of the right side muscles indicated that HS, TA, and QC muscles were significantly higher in comparison to GM. At the same time, the left side muscles showed that the TA was significantly higher in comparison to HS, QC, and GM.

\section{Discussion:}

The present study was designed to compare the EMG-RMS power values produced by lower limb right and left side muscles (QC, HS, TA, GM) during the preparatory phase of performing four heading skills from: standing, running, jumping, and running/ jumping. This was mainly done in order to assess the importance of each of the designated muscles in performing each of the four heading skills.

The overall results of right and left side muscles indicatied that the latter muscles were significantly higher than the former for heading from running/jumping and jumping, while no sgnificat differences were found for heading from standing and running. These results occurred in accordance with the demanding effort required from these muscles in accordance with each heading skill. It is a natural result, because force is directly proportional to acieration ( $\mathrm{F}=\mathrm{MA})$, if demanding on acceleration increases force also increases.

In heading from standing and running, feet during the preparatory phase should be in 
contact with the ground to form a good base, and should also be staggered, to provide balance when the upper body arches backwards as the ball arrives. The feet should be spaced apart, forming a good base. The feet should also be staggered, to provide balance when the upper body arches backwards as the ball arrives. The trunk of the body should snap forward to give power to the header as the forehead contacts the ball (8). Therefore, no significant result was found between right and left side muscles for either heading from standing or running.

The takeoff for heading from jumping is mostly one-footed takeoff (8). The critical element in heading from jump and running Jump is that the knee and ankle of the take off leg should push upward to generate vertical lift. The action of the non-takeoff leg should swing forward and high. The upper body should be arch backwards at the point of takeoff and, when maximum height is reached, the body should leaning forward. The momentum of reaching back in a snapping motion propels the upper body forward, and the energy generated puts power behind the header. The head and neck should be tense as the forehead meets the ball $(4,13)$. Therefore, the significant increase of power for left side muscles could be due the increase in the number of subjects using the left leg as a take-off leg. This may be a fault in this study because the takeoff leg should be similar for all subjects.

ANOVAs revealed significant differences existed among the four muscles, even for right or left side muscles for all heading skills ( $p>0.000)$. Tukey tests indicated that TA, HS, and QC muscles were comparable in their production of the EMG-RMS effort at the right side for all heading skills and significantly higher in comparison to GM. While the left side the TA muscle was significatly higher in comparison to the other muscles.

For all heading skills, the above results indicated that EMG-RMS effort exerted by HS and QC muscles were abuot equal either at right or left side, while the GM muscles exerted the least active in both sides.

The relative contribution of the gastrocnemius muscle to may be brought about by a decrease in power required from this muscles in performing these skills. Several authors have shown different excitation patterns for and gastrocnemius muscles in response to cadence manipulation during cycling (7), gastrocnemius operated over a narrower range of operating lengths but at a higher range of shortening velocity than soleus. The higher range of velocity may have resulted in the need for a relatively higher excitation, as indicated by the integrated EMG, as the muscle was working at a different range on its force-velocity curve. During the recovery portion of the pedalling cycle (5).

Research evidences indicated that the most active lower leg muscle is actually the TA, a strip of sinew which runs down the shin to the inside edge of the foot and pulls the foot inward and upward. During running, the tibialis anterior contracts forcefully about twice as often as any of the other four muscles, so it is probably the muscle most prone to fatigue $(2,6,12)$. Thus, it is critical for soccer players to make their tibialis anterior muscles as strong and fatigue proof as possible. Several previous studies have investigated the activity of selected extrinsic muscles in the lower leg they found increase in TA muscle EMG activity during walking $(1,2,6,8)$.

Finally, it should be noted that TA which dorsiflexes the foot, is antagonistic to the GM and soleus muscles, which plantar flex the foot (2), and TA aides in the activities of walking, running, jumping, hiking, kicking a ball, or any activity that requires moving the leg or keeping the leg vertical.

\section{References:}

1. Carley, P. EMG muscle activity and subjective influences of an insole program in the manufacturing environment. AmericanInternationalCollege, Springfield, MA, USA; DiSanto, A. http://www.megacomfort.ca/megacomfortresearch-studies.aspx.

2. Duquette AM, Andrews DM,: Tibialis anterior muscle fatigue leads to changes in tibial axial acceleration after impact when ankle dorsiflexion angles are visually controlled. Human Movement Science 2010;29:567-577. 
3. Football Science. Vo1.5, 7-17, 2008 http://www.jss£net/home.html.

4. Lees A, Nolan L. Biomechanics of Soccer A Review. Journal of Sports Sciences 1998;16: 211-234.

5. Nilsson $\mathbf{J}$, Thorstensson A and Halbertsma $\mathbf{J}$ (1985) Changes in leg movements and muscle activity with speed of locomotion and mode of progression in humans. Acta Physiologica Scandinavica 123;457-475.

6. Qaqish , J.H., McLean, S.M.. Foot type and tibialis anterior muscle activity during the stance phase of gait: A pilot study International Journal of Physiotherapy and Rehabilitation, October 2010, Vol. 1, Issue 1, 19-29.

7. Sanderson, David J., Martin P.E., Honeyman G., Keefer, J. Gastrocnemius and soleus muscle length, velocity, and EMG responses to changes in pedalling cadence Journal of Electromyography and Kinesiology xxx (2005).

8. Santello, M., McDonagh, M. J., \& Challis, J. H. (2001). Visual and non-visual control of landing movements in humans. The Journal of Physiology, 537(Pt 1), 313-327.
9. Schmitt D M, J Hertel, T A Evans, L C Olmsted, M Putukian Effect of an acute bout of soccer heading on postural control and selfreported concussion symptoms. International Journal of Sports Medicine, 2004, Volume: 25, Issue: 5, Pages: 326-331.

10. Shewchenko, N., et al. Heading in football. Part 2: biomechanics of ball heading and head response. Br J Sports Med, 2005. 39 Suppl 1: p. i26-32.

11. Sport News. Soccer Heading Techniques http://toponsports.blogspot.com/2008/03/soccerheading-techniques.html.

12. Stoquart G, Detrembleur C, Lejeune $T$ (2008) Effect of speed on kinematic, kinetic, electromyographic and energetic reference values during treadmill walking. Clinical neurophysiology 38, 105-116.

13. Sunamf, Shunsuke \& Maruyama ,Takeo. Motion and EMG Analysis of Soccer-ball Heading for the Lateral Direction. Football Science Vo1.5, 7-17, 2008. 